\title{
Sperm morphology, swimming velocity, and longevity in the house sparrow Passer domesticus
}

\author{
Fabrice Helfenstein • Murielle Podevin • Heinz Richner
}

Received: 10 July 2009 /Revised: 17 September 2009 /Accepted: 18 September 2009/Published online: 17 October 2009

(C) Springer-Verlag 2009

\begin{abstract}
Sperm competition exerts strong selection on males to produce spermatozoa with an optimal morphology that maximizes their fertilization success. Long sperm were first suggested to be favored because they should swim faster. However, studies that investigated the relationship between sperm length and sperm competitive ability or sperm swimming velocity yielded contradictory results. More recently, ratios of the different sections of a spermatozoon (the head, midpiece, and flagellum) were suggested to be more crucial in determining swimming velocity. Additionally, sperm ability to remain and survive in the female storage organs may also influence fertilization success, so that optimal sperm morphology may rather maximize sperm longevity than velocity. In this study, we investigated how sperm morphology is related to sperm velocity and sperm longevity in the house sparrow Passer domesticus. Sperm velocity was found to be correlated with head/flagellum ratio. Sperm with small heads relative to their flagellum showed higher swimming velocity. Additionally, shorter sperm were found to live longer. Finally, we found sperm morphological traits to vary substantially within males and the head/flagellum ratio to be unrelated to total sperm length. We discuss the hypothesis that the substantial within-male variation in sperm morphology reflects a male strategy to produce a diversity of sperm from long, fast-swimming to short, long-living sperm to maximize their fertilization success in a context of sperm competition.
\end{abstract}

Communicated by R. Gibson

F. Helfenstein $(\triangle) \cdot M$. Podevin $\cdot$ H. Richner

Evolutionary Ecology Group, Institute of Ecology and Evolution,

University of Bern, Baltzerstrasse 6,

3012 Bern, Switzerland

e-mail: fabrice.helfenstein@free.fr
Keywords Passer domesticus · Sperm flagellum . Sperm length · Sperm longevity $\cdot$ Sperm midpiece . Sperm morphology $\cdot$ Sperm velocity $\cdot$ Sperm competition

\section{Introduction}

It is now well established that sperm competition, defined as the competition between sperm of different males for the fertilization of a given set of ova (Parker 1970), exerts strong selection on males and drives the evolution of sperm traits that maximize their fertilizing potential and likelihood of paternity (Pizzari and Parker 2009). Despite recent studies attempting to identify the sperm characteristics that yield higher fertilization success, no clear picture can be drawn so far. In particular, the functional links between sperm morphology and sperm velocity and longevity still remain unclear.

Sperm velocity is known to be a major determinant of sperm competitive success (Pizzari and Parker 2009), with faster sperm being better able to outcompete rival sperm in the "race" to the ovum. Gage et al. (2004) reported that relative velocity of the sperm of two rival males was positively related to sperm competition success in the Atlantic salmon (Salmo salar). In the domestic fowl (Gallus domesticus), sperm mobility determines male fertility and sperm competitive ability (Birkhead et al. 1999; Froman et al. 1999). Gomendio and Roldan (2008) suggested that descriptors of sperm swimming velocity may indeed reflect sperm ability to surmount the barriers in the female reproductive tract and to escape the hostile female environment (Birkhead et al. 1993). It is worth bearing in mind, however, that in many internally fertilizing species, sperm transport within the female is almost exclusively determined by female contractions rather than by sperm movements (Birkhead and Brillard 2007). 
More than 15 years ago, Gomendio and Roldan (1991) were the first to propose that sperm swimming velocity may be functionally related to sperm size, with longer sperm swimming faster. Evidence in support of this hypothesis exists at the interspecific level in mammals (Gomendio and Roldan 1991, 2008) and birds (Lüpold et al. 2009a). At the intraspecific level, though, the few studies investigating the relationship between sperm size and sperm velocity yielded inconsistent results. In the nematode Caenorhabditis elegans, which produces aflagellate amoeboid sperm, LaMunyon and Ward (1998) found larger sperm to move faster. A study on sand martins Riparia riparia found shorter sperm to swim faster (Helfenstein et al. 2008), while in the zebra finch Taenopygia guttata, sperm morphology was found to be positively related to and genetically linked with sperm velocity (Mossman et al. 2009). Overall, however, most studies found no association between sperm size and sperm velocity (e.g., Gage et al. 2002; Minoretti and Baur 2006; Pitcher et al. 2007). As a matter of fact, recent theoretical developments suggested that ratios between the different sections of a spermatozoon (the head, midpiece, and flagellum) may have a greater impact on sperm velocity than total sperm length. Humphries et al. (2008) proposed that the ratio between the length of the sperm head and the length of the flagellum should be determinant, because the head produces a drag proportional to its length that needs to be compensated by the thrust of the flagellum. Additionally, midpiece length has also been hypothesized to be associated with greater energy supply through ATP production in the mitochondria (Cardullo and Baltz 1991). Although glycolysis occurs in the flagellum, the spermatozoon propeller, the midpiece may provide a significant portion of the energy used for propulsion (Anderson and Dixson 2002). These hypotheses led to the predictions that, first, there should be a positive relationship between the head length and the flagellum length on the one hand and between the midpiece length and the flagellum length on the other hand, and that, second, head/midpiece and midpiece/flagellum ratios should be related to sperm velocity (Cardullo and Baltz 1991). In support of these theoretical developments, Cardullo and Baltz (1991) and Immler and Birkhead (2007) found positive allometries between the midpiece and the flagellum across mammals and birds, respectively. In addition, Lüpold et al. (2009b) found, at the interspecific level, a negative relationship between head length and velocity and a positive relationship between flagellum length and velocity. Yet, ratios between the different sections of the sperm had the same impact on sperm velocity as absolute lengths. Moreover, against the prediction that midpiece length determines sperm velocity, Malo et al. (2006) found in the red deer Cervus elaphus hispanicus that sperm with long midpieces are slower, whereas sperm with long heads and long flagella have greater velocity.
Like the relationship between sperm size and sperm velocity, the relationship between sperm size and sperm longevity remains unclear so far. Stockley et al. (1997) found that sperm longevity decreases with sperm length across species of fish. In a comparative study in pheasants, Immler et al. (2007) also found that sperm longevity, measured as the number of days females store sperm before fertilization, was negatively correlated with sperm size. Inversely, in a study on sand martins $R$. riparia, Helfenstein et al. (2008) found longer sperm (i.e., sperm with longer midpiece, midpiece length being correlated with total sperm length; Kleven et al. 2009) to swim more slowly than shorter sperm but to live longer, and the authors suggested that sperm velocity and sperm longevity may be traded off according to the male ability to access and copulate with fertile females as well as the timing of the copulation relative to fertilization.

Our aims in this study were threefold. First, we investigated the relationship between the length of the different sections of a spermatozoon between and within ejaculates of male house sparrows Passer domesticus. Second, we were interested in the relationship between sperm morphology (absolute length and ratios of the different sections) and sperm velocity and longevity. Our last goal was to quantify variance in sperm morphological traits between males, within males, and between repeated measures of the same sperm. The house sparrow is a well-known species frequently used in studies of sexual selection processes (Nakagawa et al. 2007). It is also an appropriate model when studying sperm traits in a context of sperm competition because females regularly engage in extrapair copulations and produce extrapair offspring (Nakagawa et al. 2007), so males are under strong selective pressure to maximize their fertilization success and ensure their paternity.

\section{Materials and methods}

Study species and sampling procedure

This study was conducted on a house sparrow $P$. domesticus population between 6 May and 21 May 2008 in the Nationales Pferdezentrum, a horse breeding center in Bern, Switzerland. Male house sparrows were captured using mist nets placed across the stables' doors. We collected sperm samples $(1-2 \mu \mathrm{l})$ using cloacal massages (Wolfson 1952). The samples were immediately added to $100 \mu$ of prewarmed $\left(40^{\circ} \mathrm{C}\right)$ Dulbecco's modified Eagle's medium (4,500 mg glucose/l, $110 \mathrm{mg}$ sodium pyruvate/1, $4 \mathrm{mM} \mathrm{L}$ glutamine, Sigma Aldrich). A 5- $\mu$ l sample was then loaded within a prewarmed $\left(40^{\circ} \mathrm{C}\right) 80$ - $\mu$ m-deep dual-sided sperm analysis chamber (Hamilton Thorne Research, Beverly, MA, USA), and sperm motion was recorded for $10 \mathrm{~min}$ at $40^{\circ} \mathrm{C}$ 
with a video camera mounted to the microscope at $\times 100$ magnification under dark-field condition generated with a Ph2 annular phase ring. Videos were directly stored on a computer hard drive via a video acquisition kit (Dazzle video creator $720 \times 576$ pixels, $25 \mathrm{~Hz}$ frame rate, Pinnacle).

\section{Sperm velocity and sperm longevity}

Video recordings of sperm motion were analyzed using a CASA plug-in to the free software ImageJ (ImageJ 1.42, Wayne Rasband, http://rsb.info.nih.gov/ij) developed by Wilson-Leedy and Ingermann (2007). Settings were adapted to passerine sperm swimming behavior (straight trajectory) and to our recording conditions (no bulk flow). Due to technical problems with some of the recordings, we discarded the first $25 \mathrm{~s}$. Sperm motion was analyzed for $2 \mathrm{~s}$ after 25, 85, and $145 \mathrm{~s}$ of video recording. Preliminary analyses showed that a 2-s sampling interval maximized the detectability of moving sperm and minimized the number of sperm entering the field of the camera, which would inflate the percentage of motile sperm. The minimum size of a sperm cell was set to 2 pixels and its maximum size to 50 pixels to exclude dust particles from the analysis. The minimum track time was $0.8 \mathrm{~s}$, and the search radius was set to 8 pixels, which minimized the risk of counting a moving particle as a motile sperm or to merge crossing sperm into a single track. Sperm cells having a straight line velocity $(\mathrm{VSL})<5 \mu \mathrm{m} / \mathrm{s}$, a curvilinear velocity $(\mathrm{VCL})<10 \mu \mathrm{m} / \mathrm{s}$, or an average path velocity $(\mathrm{VAP})<10 \mu \mathrm{m} / \mathrm{s}$ were counted as immotile.

The CASA plug-in recorded mean values for the percentage of motile sperm, VCL (total distance traveled, micrometers per second), VAP (smoothed path using roaming average, micrometers per second), and VSL (distance from origin to the furthest point reached during the measured time period, micrometers per second). We also recorded the number of sperm cells detected by the CASA plug-in because moving sperm are likely to interfere more with each other at higher densities, a parameter we could not control. Sperm velocity has previously been described using VSL, VAP, or VCL or a combination of these parameters (Holt et al. 1997; Froman and Feltmann 2000; Gage et al. 2004; Pizzari et al. 2004). In our samples, these parameters were strongly correlated with each other $(r, 0.93-0.998$; all $P<0.0001)$. Therefore, we used an index of sperm velocity based on the first principal component of principal component analysis (PCA) conducted on VSL, VAP, VCL, and the number of sperm cells detected by the CASA. PC1 explained $77.1 \%$ of the variance and was strongly positively correlated with VSL, VCL, and VAP (all $r>0.96, P<0.0001)$ but independent of the sperm density in the sample (all $|r|<0.23, P>0.11$ ).

We took three measures of VSL, VCL, and VAP after 25,85 , and $145 \mathrm{~s}$ of video recording. This allowed us to derive sperm longevity from statistical models as the rate at which the percentage of motile, living sperm declines with time. This also allowed us to monitor the dynamics of sperm velocity over time. Technical adjustments caused the delay between the collection of the ejaculate and the start of the video recording to vary among individuals (mean \pm standard error (SE), $101.25 \pm 4.30 \mathrm{~s}$; range, 30-200 s). This delay was recorded, and we used the total time since sample collection (i.e., the duration of sample processing and recording duration to the start of analysis) as our time variable in all models.

\section{Sperm morphology}

A second sample of sperm was collected immediately after the first and stored in $100 \mu \mathrm{l}$ formalin (5\%) for later measurement of sperm morphometry. In the laboratory, a drop of sperm/ formalin solution was deposited on a slide and air-dried. The slide was then rinsed with distilled water, fixed with Glycogel (Roth AG), and covered with a cover slip. Photographs of spermatozoa were taken with an Olympus E-520 digital camera (10 megapixels) mounted on an Olympus microscope at $\times 400$ magnification under phase contrast generated with a $\mathrm{Ph} 2$ annular phase ring. From these pictures, we selected ten intact spermatozoa (no broken tail, midpiece correctly coiled around the flagellum) per individual. All spermatozoa were sketched in a notebook to enable a second measurement later. All pictures were analyzed by MP using ImageJ at a resolution of 8,267 pixels $/ \mathrm{mm}$.

For each spermatozoon, we recorded its total length, the length of its head, midpiece (straight length of the part curled around the flagellum), and flagellum. In order to assess the repeatability of the method, three spermatozoa from 20 males were measured a second time in a distinct measurement batch and without knowledge of the values previously obtained for those sperm. In addition to absolute lengths, we also computed the three ratios used in previous studies and predicted to influence sperm velocity: the head/midpiece ratio, the head/ flagellum ratio, and the midpiece/flagellum ratio.

\section{Statistical analyses}

We used random models to partition total variance into variance between males, within males, and between repeated measures of the same spermatozoon and estimate within-male and within-sperm (measurement) repeatability. We used generalized linear mixed models with restricted maximum likelihood parameter estimation function (REML-GLMMs) with male identity as the random factor to investigate the within-male, within-ejaculate relationships between head size, midpiece size, and flagellum size. We used REML-GLMMs for repeated measures to investigate how sperm morphological traits are related to sperm longevity and to the temporal dynamics of sperm velocity. Male identity was defined as the 
random subject. Intrasubject variance was modeled using an unstructured $R$ matrix, which accounts for heteroskedasticity between measurement times and intrasubject correlation between measures (Littell et al. 2006). We used time as a continuous variable because sperm motility and sperm velocity regularly declined with time. We present terms with their estimates $\beta( \pm \mathrm{SE})$ and two-tailed type-III $F$ and $P$ values. Degrees of freedom of fixed effects were computed using the between-/within-subject portions method (Littell et al. 2006). Modeling assumptions were validated by thorough visual inspection of the studentized residuals plotted against the predicted values and by testing the residuals' normality. The models' robustness was also checked by removing the most extreme values. Graphical representations of the interactions were built using the predicted values derived from the model. All analyses were conducted using the SAS software version 9.1 (SAS ${ }^{\circledR} 2003$ ).

\section{Results}

We obtained data on sperm morphology from 49 male house sparrows. Total sperm length and the length of the different sperm sections are summarized in Table 1. In general, we found measurement error to be low and repeatability of the measures to be high, ranging from 0.74 to 0.97 (Table 2). We also found significant between-male variation (Fig. 1) and significant within-male repeatability for all traits measured. However, within-male variance was always substantial (Fig. 1), ranging from $40 \%$ (total sperm length) to $76 \%$ (head length) of the total variance, and within-male repeatability, although statistically significant, was low for the head and midpiece length (0.24 and 0.32 , respectively; Table 2$)$.

Sperm morphology and sperm swimming speed

We obtained data on both sperm morphology and sperm motility from 48 males. Among the different lengths and section ratios investigated, only the head/flagellum ratio was found to be related to sperm velocity. Spermatozoa having shorter heads relative to their flagellum showed a higher swimming velocity (Table 3; Fig. 2; all other traits, absolute lengths, or section ratios: all $F<2.28, P>0.13$ ). Adding a quadratic term for sperm traits did not improve the fit of the models.
Sperm morphology and sperm longevity

We found total sperm length to be associated with sperm longevity (significant interaction between total sperm length and time; Table 4). In other words, ejaculates with long sperm showed a higher percentage of motile sperm soon after ejaculate collection (ca. $65 \%$ motile sperm) but those sperm died rapidly as time passed (ca. 15\% motile sperm after $345 \mathrm{~s}$ ). Conversely, ejaculates containing short sperm showed moderate motility (ca. $40 \%$ motile sperm) but those sperm showed higher longevity (Fig. 3). We found a similar relationship between flagellum length and longevity, although the correlation was less tight (Table 4) and likely due to the correlation between total sperm length and flagellum length across males $\left(F_{1,47}=367.15, P<\right.$ 0.0001). None of the other traits (absolute lengths or section ratios) showed significant association with sperm longevity (all $F<2.75, P>0.10$ ). Adding a quadratic term for sperm traits did not improve the fit of the models.

\section{Relationships between sperm morphological traits}

Across males, we found no significant relationship between the size of the head and the size of the midpiece or the size of the flagellum (all $F_{1,47}<0.98, P>0.33$ ). However, we found a positive linear relationship between the midpiece and the flagellum length (best-fitted model: midpiece length $=27.57+0.46 \times$ flagellum length; $F_{1,}{ }_{47}=26.2, P<$ 0.0001; other models such as Log-Log did not improve the fit of the relationship).

Within ejaculates, contrary to our expectations, we found flagellum length and midpiece length to be negatively correlated with head length (best-fitted REML-GLMMs: flagellum length $=90.67-0.50 \times$ head length; $F_{1,440}=48.58$, $P<0.0001 ;$ midpiece length $=74.63-0.57 \times$ head length; $F_{1,440}=51.64, P<0.0001$; other models such as Log-Log did not improve the fit of the relationship). However, midpiece length was positively correlated with flagellum length (best-fitted REML-GLMM: midpiece length $=24.96+$ $0.49 \times$ flagellum length; $F_{1}, 440=132.1, P<0.0001$; other models such as Log-Log did not improve the fit of the relationship).

To have a better understanding of the relationships between head/flagellum ratio and swimming velocity on the one hand and total sperm length and sperm longevity on
Table 1 Variation in total sperm length and the length of the different sperm sections (micrometers) among a sample of ten sperm for each of 49 male house sparrows

\begin{tabular}{lccccc}
\hline & Mean & Standard deviation & Median & Minimum & Maximum \\
\hline Total sperm length & 98.9 & 3.7 & 99 & 86 & 109 \\
Head length & 15.8 & 1.8 & 16 & 7 & 22 \\
Midpiece length & 65.6 & 3.4 & 66 & 51 & 79 \\
Flagellum length & 82.8 & 3.5 & 83 & 70 & 94 \\
\hline
\end{tabular}


Table 2 Partitioning of the variance in sperm morphological traits into measurement error, within-male variance, and between-male variance

\begin{tabular}{|c|c|c|c|c|c|c|}
\hline & \multicolumn{6}{|c|}{ Sources of variance } \\
\hline & $\begin{array}{l}\text { Between and } \\
\text { within males }\end{array}$ & $\begin{array}{l}\text { Measurement } \\
\text { error }\end{array}$ & $\begin{array}{l}\text { Between } \\
\text { males }\end{array}$ & Within males & $\begin{array}{l}\text { Measurement } \\
\text { repeatability }\end{array}$ & $\begin{array}{l}\text { Within-male } \\
\text { repeatability }\end{array}$ \\
\hline Total sperm length & & & & & 0.97 & 0.60 \\
\hline Variance estimate $\pm \mathrm{SE}$ & $15.41 \pm 2.89$ & $0.53 \pm 0.10$ & $8.34 \pm 1.82$ & $5.57 \pm 0.37$ & & \\
\hline Head length & & & & & 0.85 & 0.24 \\
\hline Variance estimate $\pm \mathrm{SE}$ & $2.87 \pm 0.57$ & $0.53 \pm 0.10$ & $0.72 \pm 0.20$ & $2.32 \pm 0.16$ & & \\
\hline Midpiece length & & & & & 0.74 & 0.32 \\
\hline Variance estimate $\pm \mathrm{SE}$ & $10.36 \pm 2.26$ & $3.61 \pm 0.66$ & $3.68 \pm 0.92$ & $7.92 \pm 0.53$ & & \\
\hline Flagellum length & & & & & 0.92 & 0.52 \\
\hline Variance estimate $\pm \mathrm{SE}$ & $12.51 \pm 2.41$ & $1.14 \pm 0.21$ & $6.61 \pm 1.47$ & $6.09 \pm 0.41$ & & \\
\hline
\end{tabular}

The variance due to measurement error was assessed using the 20 males of which we measured three spermatozoa twice. The within- and between-male variance were assessed using all 490 sperm measures, i.e., ten sperm per male for 49 males. All morphological traits showed high, significant measurement repeatability (all $\left.F_{59,60}>29.01, P<0.0001\right)$ and moderate to low, statistically significant within-male repeatability (all $\left.F_{48,441}>4.12, P<0.0001\right)$

the other hand, we explored the relationship between total sperm length and head/flagellum ratio. These two traits were correlated neither across nor within males $\left(F_{1,47}=\right.$ $0.26, P=0.61 ; F_{1,440}<0.01, P=0.93$, respectively).

\section{Discussion}

We found substantial within-male variance and significant but moderate to low within-male repeatability in the length
Fig. 1 Within- and betweenmale variance in sperm morphological traits based on ten spermatozoa for each of 49 males. Dots represent individual mean lengths ( \pm standard deviation) ranked in order of magnitude
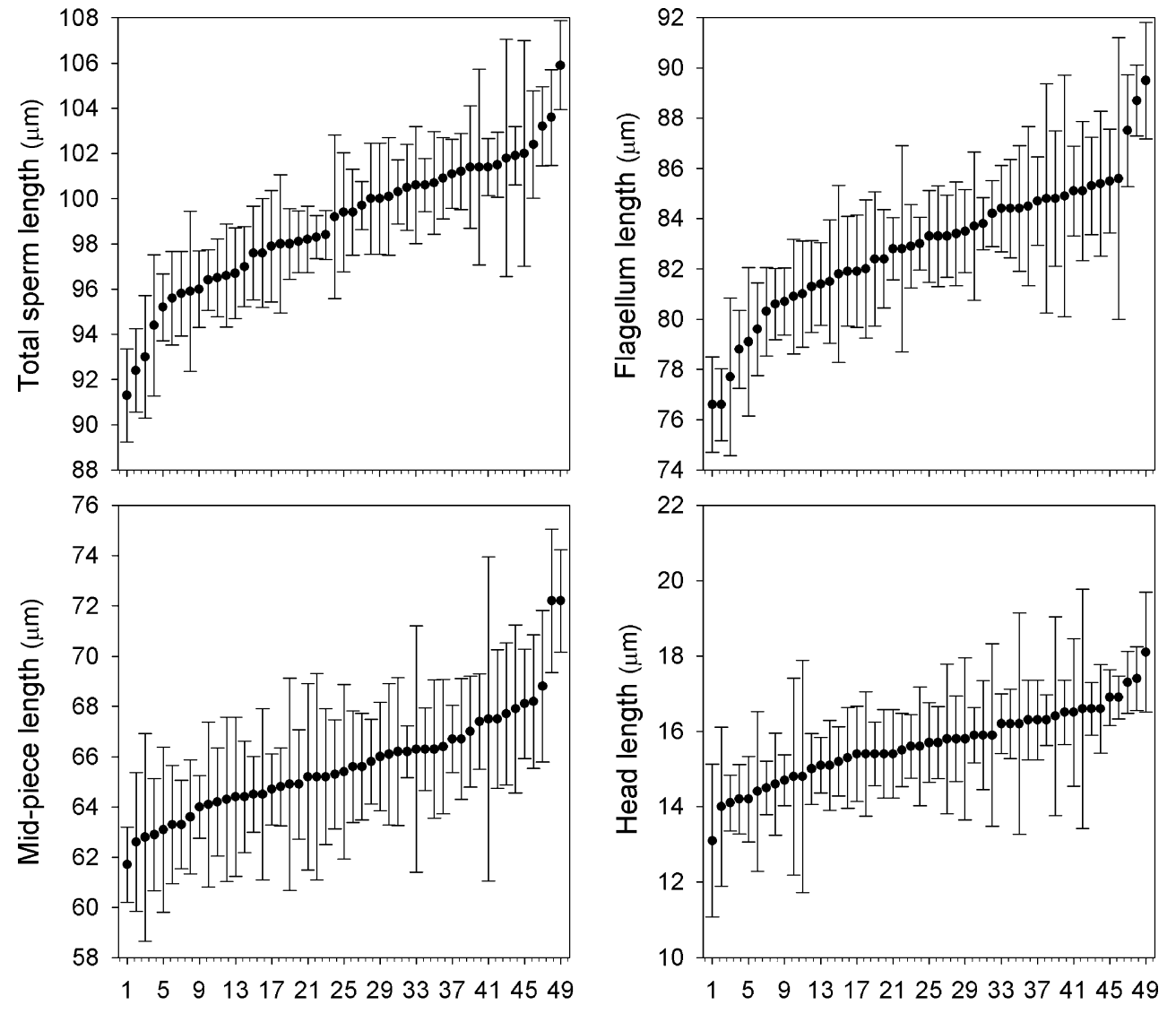

Arbitrary male number 
Table 3 REML-GLMM investing the temporal dynamics of sperm velocity in relation to the head/flagellum ratio

\begin{tabular}{llll}
\hline Independent variable & Estimate \pm SE & $F_{d f}$ & $P$ \\
\hline Intercept & $4.78 \pm 1.72$ & - & - \\
Time & $-0.0054 \pm 0.0008$ & $43.55_{1,46}$ & $<0.0001$ \\
Head/flagellum ratio & $-19.97 \pm 9.06$ & $4.87_{1,46}$ & 0.032 \\
Time $\times$ head/flagellum ratio & $-0.0007 \pm 0.0643$ & $<0.01_{1,46}$ & 0.99 \\
\hline
\end{tabular}

of the sperm morphological traits we measured. We also found spermatozoa with short heads relative to their flagellum to swim faster and short sperm to live longer. However, head/flagellum ratio and total sperm length were correlated neither across nor within males. Hence, male house sparrows seem to produce a mixture of long and short sperm with small or large head/flagellum ratios.

In this study, we were able to assess measurement error and within- and between-male variance in sperm morphological traits. Measurement error was generally low, although it reached $26 \%$ for midpiece length, illustrating how difficult it may be to identify certain sections of a spermatozoon even under good conditions $(\times 400$ magnifications, phase contrast). We found substantial within-male variance for all sperm morphological traits, ranging from $40 \%$ (total sperm length) to $76 \%$ (head length) of the total variance. This contrasts with results found in three species of birds where within-male repeatability of sperm length was high (zebra finch T. guttata, 0.87; Birkhead and Fletcher 1995; bluethroat Luscinia svecica, 0.73; and willow warbler Phylloscopus trochilus, 0.79; Laskemoen et al. 2007). Our results are, however, comparable to what has been found in the red deer C. elaphus hispanicus (Malo et al. 2006), where, although repeatability was statistically significant, within-male variance in total sperm length accounted for $43 \%$ of the total variance. These results also

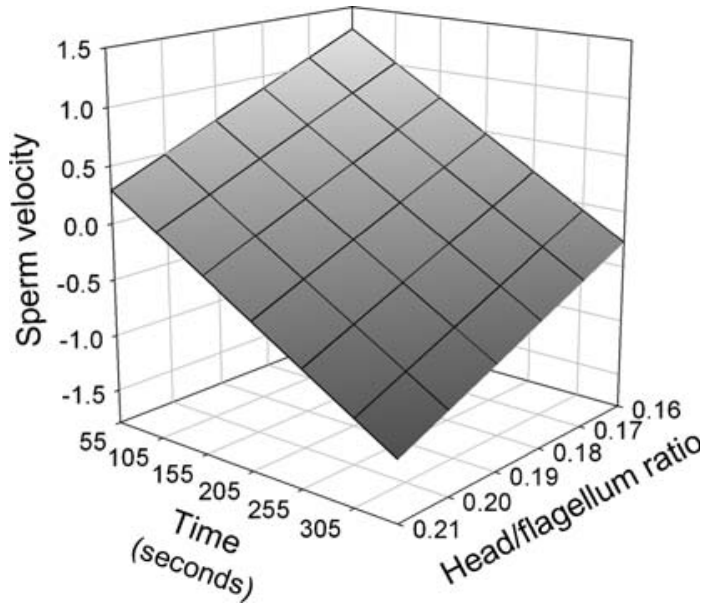

Fig. 2 Sperm swimming velocity (PCA scores, larger values indicate higher swimming velocity, see "Materials and methods" section) as a function of head/flagellum ratio and time after ejaculation. Data are predicted values from a REML-GLMM contradict theoretical developments predicting that postcopulatory sexual selection should result in stabilizing selection, disfavoring extreme sperm morphologies, and reducing variation in sperm size (Calhim et al. 2007; Kleven et al. 2009). The substantial within-male variance we found in house sparrows raises two questions: How is variance in sperm morphology maintained and has sperm morphology evolved toward a single optimum that maximizes male fertilization success?

A first explanation would be that spermatogenesis is poorly controlled, and postcopulatory selection is low in this house sparrow population. It is, however, unlikely because female house sparrows have been repeatedly shown, in different populations, to regularly engage in extrapair copulations and to produce extrapair offspring (reviewed in Nakagawa et al. 2007), and sperm competition is intense in this species. Alternatively, males may produce a variety of sperm with different sizes and morphologies as a strategy to maximize their fertilization success. Most studies have focused on the functional relationship between sperm morphology and sperm velocity. Longer sperm or sperm with optimal section ratios (e.g., head/flagellum or midpiece/flagellum) have been hypothesized to swim faster and to be selected via sperm competition (Gomendio and Roldan 1991; Briskie and Montgomerie 1992; Briskie et al. 1997; Gomendio and Roldan 2008; Lüpold et al. 2009b). However, the morphology and physiology of the female reproductive tract and female use of stored sperm may also have a prominent impact on male fertilization success (Briskie et al. 1997; Calhim et al. 2007; García-Gonzáles and Simmons 2007; Humphries et al. 2008). In line with this hypothesis, Immler et al. (2007) found that spermatozoa are shorter in species where sperm are stored by females for a longer period. Males generally have incomplete knowledge of female fertility and therefore do not know exactly when their sperm will be used for fertilization. If swimming velocity and longevity are traded off or if they are functionally linked to different sperm morphological traits, it may become adaptive to produce a variety of sperm morphologies that may maximize fertilization success when inseminated at different times relative to the fertilization of the ovum.

Our results accord with this hypothesis. We found that sperm with small heads relative to their flagellum had significantly higher swimming velocity, which is consistent 
Table 4 GLM investigating the temporal dynamics of sperm motility in relation to total sperm length and flagellum length

\begin{tabular}{lcllllll}
\hline Independent variable & Estimate $\pm \mathrm{SE}$ & $F_{d f}$ & $P$ & Independent variable & Estimate $\pm \mathrm{SE}$ & $F_{d f}$ & $P$ \\
\hline Intercept & $-2.03 \pm 1.02$ & - & - & Intercept & $-1.27 \pm 0.97$ & - \\
Time & $0.010 \pm 0.004$ & $5.42_{1,46}$ & 0.024 & Time & $0.007 \pm 0.004$ & $3.52_{1,46}$ & 0.07 \\
Total sperm length & $0.026 \pm 0.010$ & $6.52_{1,46}$ & 0.014 & Flagellum length & $0.022 \pm 0.012$ & $3.61_{1,46}$ & 0.06 \\
Time $\times$ total sperm length & $-0.00011 \pm 0.00004$ & $6.47_{1,46}$ & 0.014 & Time $\times$ flagellum length & $-0.00010 \pm 0.00005$ & $4.41_{1,46}$ & 0.04 \\
\hline
\end{tabular}

with theory (Humphries et al. 2008). Sperm with a smaller head/flagellum ratio benefit from the stronger propulsion force of a relatively long flagellum and the smaller drag produced by a relatively small head. We also observed that ejaculates containing long sperm showed high motility right after ejaculation but this motility then steeply declined with time, whereas short sperm remained motile for a longer time. We found a similar result when focusing on the sperm flagellum. This is not surprising as it is highly correlated with total sperm length, the flagellum representing more than $80 \%$ of the total length. Thus, long sperm appear to be more motile but to exhaust their energy faster and have reduced longevity. Interestingly, the head/flagellum ratio and total sperm length were not correlated, neither across nor within males. Moreover, within-male variances in total sperm length and in the size of the head and the size of the flagellum were substantial $(40 \%, 76 \%$, and $48 \%$, respectively). This suggests that a male's ejaculate may comprise a mixture of long and short spermatozoa with small or large head/flagellum ratios.

Female birds store sperm in sperm storage tubules (SSTs), and Froman (2003) proposed that sperm remain in the SSTs by swimming against a flow generated by secretion in the distal end of the SSTs. Sperm longevity and ability to swim

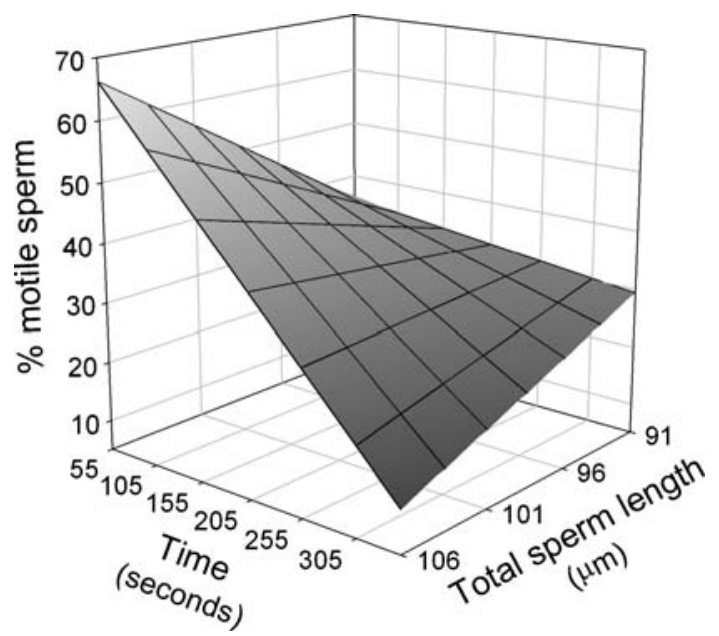

Fig. 3 Percentage of motile sperm (proportion square-root transformed $\times 100$ ) as a function of total sperm length (micrometers) and time after ejaculation. Data are predicted values from a REMLGLMM including time, total sperm length, and the interaction between time and sperm length above a certain threshold would then determine for how long sperm are stored in the SSTs. Nevertheless, when sperm are lost from the SSTs, they are passively transported to the infundibulum, the site of fertilization (Birkhead and Brillard 2007). Under this scenario and in a context of sperm competition, fast sperm may be able to enter the SSTs more quickly and before rival sperm, thus gaining advantage by the number. Although their swimming velocity may decline steeply, these sperm may still ensure high paternity if fertilization occurs shortly after insemination. On the contrary, long-living sperm may not swim fast but may remain longer in the SSTs, gaining advantage as short-living sperm are lost from the SSTs (Pizzari et al. 2008a). Long-living sperm may thus ensure high paternity when fertilization occurs several hours to several days after insemination (Immler et al. 2007).

If males may benefit in producing a variety of sperm morphs, which would explain within-male variance in sperm morphology, between-male variation may well be determined by individual mating strategies and ability to access fertile females and obtain copulations. Trade-offs may exist between investing in somatic traits that enhance male attractiveness (secondary sexual characters), social status (fighting ability) and/or paternity insurance (mate guarding), and semen traits that confer competitive advantages to the gametes (Parker 1998). Studies in species where males exhibit discrete mating roles or where a strong social hierarchy determines mating opportunities, resident, or dominant males have been shown to produce sperm with lower swimming velocity than satellite or subdominant males (Vladić and Järvi 2001; Froman et al. 2002; Neff et al. 2003; Gage et al. 2004; Rudolfsen et al. 2006; Cornwallis and Birkhead 2007; Pizzari et al. 2007). Male house sparrows exhibit a melanin-based badge of status, and social rank may determine a male's access to fertile females (Nakagawa et al. 2007). Less attractive and/or low-ranking males may prioritize sperm production over other somatic functions, thus producing between-male variance in sperm morphological traits, swimming velocity, and sperm longevity.

We have no information on male age or sexual rest. These two parameters may be related to social status and to male ability to access females and may thus also determine reproductive strategies (Wedell et al. 2002; Dean et al. 2007). Hence, sperm morphology, sperm velocity, and the relationships between sperm traits may vary according to 
male age and sexual activity. In addition, recent studies have shown that sperm quality decreases as males get older (Dean et al. 2007; Pizzari et al. 2008b; Møller et al. 2009), and male age may thus confound the relationships between sperm morphology, velocity, and motility through senescence processes. How social status, male age, and male sexual activity relate to sperm characteristics in the house sparrow still remains to be investigated.

In this study, we conducted multiple analyses to explore the potential relationships between a number of sperm morphological traits and sperm velocity and longevity. This increases the risk of making type I errors, i.e., to find spurious, statistically significant correlations. Corrections for multiple tests such as Bonferroni procedures reduce such a risk, but have drawbacks (i.e., increased type II error; Nakagawa 2004). We thus did not correct for test multiplicity. Nevertheless, the possibility that some of our results emerged from a type I error should be kept in mind, and such a study should be repeated on an independent sample to confirm our results.

Acknowledgments We are very grateful to Dr. Beat Wampfler for allowing us to work in the Nationales Pferdezentrum Bern. The authors would also like to thank the two anonymous referees for their valuable comments on the manuscript. This work was conducted under license of the Ethical Committee of the Agricultural Office of the Canton Bern and a ringing permit provided by the Agency for Environment, Forests, and Landscapes.

Authors' contributions FH designed the study, collected the data, and analyzed sperm motility; MP measured sperm; FH and MP analyzed data and wrote the paper; and HR supervised the study. This study was part of MP's bachelor's work.

\section{References}

Anderson MJ, Dixson AF (2002) Sperm competition: motility and the midpiece in primates. Nature 416:496

Birkhead TR, Brillard J-P (2007) Reproductive isolation in birds: postcopulatory prezygotic barriers. Trends Ecol Evol 22:266-272

Birkhead TR, Fletcher F (1995) Male phenotype and ejaculate quality in the zebra finch Taeniopygia guttata. Proc R Soc Lond B 262:329-334

Birkhead TR, Møller AP, Sutherland WJ (1993) Why do females make it so difficult for males to fertilize their eggs? J Theor Biol $161: 51-60$

Birkhead TR, Martinez JG, Burke T, Froman DP (1999) Sperm mobility determines the outcome of sperm competition in the domestic fowl. Proc R Soc Lond B 266:1759-1764

Briskie JV, Montgomerie R (1992) Sperm size and sperm competition in birds. Proc R Soc Lond B 247:89-95

Briskie JV, Montgomerie R, Birkhead TR (1997) The evolution of sperm size in birds. Evolution 51:937-945

Calhim S, Immler S, Birkhead TR (2007) Postcopulatory sexual selection is associated with reduced variation in sperm morphology. PLoS ONE 2:e413

Cardullo RA, Baltz JM (1991) Metabolic regulation in mammalian sperm: mitochondrial volume determines sperm length and flagellar beat frequency. Cell Motil Cytoskelet 19:180-188
Cornwallis CK, Birkhead TR (2007) Changes in sperm quality and numbers in response to experimental manipulation of male social status and female attractiveness. Am Nat 170:758-770

Dean R, Bonsall MB, Pizzari T (2007) Aging and sexual conflict. Science 316:383-384

Froman DP (2003) Deduction of a model for sperm storage in the oviduct of the domestic fowl (Gallus domesticus). Biol Reprod 69:248-253

Froman DP, Feltmann AJ (2000) Sperm mobility: phenotype in roosters (Gallus domesticus) determined by concentration of motile sperm and straight line velocity. Biol Reprod 62:303-309

Froman DP, Feltmann AJ, Rhoads ML, Kirby JD (1999) Sperm mobility: a primary determinant of fertility in the domestic fowl (Gallus domesticus). Biol Reprod 61:400-405

Froman DP, Pizzari T, Feltmann AJ, Castillo-Juarez H, Birkhead TR (2002) Sperm mobility: mechanisms of fertilizing efficiency, genetic variation and phenotypic relationship with male status in the domestic fowl, Gallus gallus domesticus. Proc R Soc Lond B 269:607-612

Gage MJG, Macfarlane C, Yeates S, Shackleton R, Parker GA (2002) Relationships between sperm morphometry and sperm motility in the Atlantic salmon. J Fish Biol 61:1528-1539

Gage MJG, Macfarlane CP, Yeates S, Ward RG, Searle JB, Parker GA (2004) Spermatozoal traits and sperm competition in Atlantic salmon: relative sperm velocity is the primary determinant of fertilization success. Curr Biol 14:44

García-Gonzáles F, Simmons LW (2007) Shorter sperm confer higher competitive fertilization success. Evolution 61:816-824

Gomendio M, Roldan ERS (1991) Sperm competition influences sperm size in mammals. Proc R Soc Lond B 243:181-185

Gomendio M, Roldan ERS (2008) Implications of diversity in sperm size and function for sperm competition and fertility. Int J Dev Biol 52:439-447

Helfenstein F, Szép T, Nagy Z, Kempenaers B, Wagner RH (2008) Between-male variation in sperm size, velocity and longevity in sand martins Riparia riparia. J Avian Biol 39:647-652

Holt C, Holt WV, Moore HDM, Reed HC, Curnock RM (1997) Objectively measured boar sperm motility parameters correlate with the outcomes of on-farm inseminations: results of two fertility trials. J Androl 18:312-323

Humphries S, Evans JP, Simmons LW (2008) Sperm competition: linking form and function. BMC Evol Biol 8:319

Immler S, Birkhead TR (2007) Sperm competition and sperm midpiece size: no consistent pattern in passerine birds. Proc $\mathrm{R}$ Soc Lond B 274:561-568

Immler S, Saint-Jalme M, Lesobre L, Sorci G, Roman Y, Birkhead TR (2007) The evolution of sperm morphometry in pheasants. J Evol Biol 20:1008-1014

Kleven O, Laskemoen T, Lifjeld JT (2009) Sperm length in sand martins Riparia riparia: a comment on Helfenstein et al. J Avian Biol 40:241-242

LaMunyon CW, Ward S (1998) Larger sperm outcompete smaller sperm in the nematode Caenorhabditis elegans. Proc R Soc Lond B 265:1997-2002

Laskemoen T, Kleven O, Fossoy F, Lifjeld JT (2007) Intraspecific variation in sperm length in two passerine species, the bluethroat Luscinia svecica and the willow warbler Phylloscopus trochilus. Ornis Fenn 84:131-139

Littell RC, Milliken GA, Stroup WW, Wolfinger RD, Schabenberger O (2006) SAS for mixed models, 2nd edn. SAS Institute, Cary

Lüpold S, Linz GM, Birkhead TR (2009a) Sperm design and variation in the New World blackbirds (Icteridae). Behav Ecol Sociobiol 63:899-909

Lüpold S, Calhim S, Immler S, Birkhead TR (2009b) Sperm morphology and sperm velocity in passerine birds. Proc R Soc Lond B 276:1175-1181 
Malo AF, Gomendio M, Garde J, Lang-Lenton B, Soler AJ, Roldan ERS (2006) Sperm design and sperm function. Biol Lett 2:246-249

Minoretti N, Baur B (2006) Among- and within-population variation in sperm quality in the simultaneously hermaphroditic land snail Arianta arbustorum. Behav Ecol Sociobiol 60:270-280

Mossman J, Slate J, Humphries S, Birkhead TR (2009) Sperm morphology and velocity are genetically codetermined in the zebra finch. Evolution 63:2730-2737

Møller AP, Mousseau TA, Rudolfsen G, Balbontín J, Marzal A, Hermosell I, De Lope F (2009) Senescent sperm performance in old male birds. J Evol Biol 22:334-344

Nakagawa S (2004) A farewell to Bonferroni: the problems of low statistical power and publication bias. Behav Ecol 15:1044-1045

Nakagawa S, Ockendon N, Gillespie DOS, Hatchwell BJ, Burke T (2007) Assessing the function of house sparrows' bib size using a flexible meta-analysis method. Behav Ecol 18:831-840

Neff BD, Fu P, Gross MR (2003) Sperm investment and alternative mating tactics in bluegill sunfish (Lepomis macrochirus). Behav Ecol 14:634-641

Parker GA (1970) Sperm competition and its evolutionary consequences in the insects. Biol Rev 45:525-567

Parker GA (1998) Sperm competition and the evolution of ejaculates: towards a theory base. In: Birkhead TR, Møller AP (eds) Sperm competition and sexual selection. Academic, San Diego, pp 3-54

Pitcher TE, Rodd FH, Rowe L (2007) Sexual colouration and sperm traits in guppies. J Fish Biol 70:165-177

Pizzari T, Parker GA (2009) Sperm competition and sperm phenotype. In: Birkhead TR, Hosken DJ, Pitnick S (eds) Sperm biology: an evolutionary perspective. Academic, San Diego, pp 207-245
Pizzari T, Jensen P, Cornwallis CK (2004) A novel test of the phenotype-linked fertility hypothesis reveals independent components of fertility. Proc R Soc Lond B 271:51-58

Pizzari T, Cornwallis CK, Froman DP (2007) Social competitiveness associated with rapid fluctuations in sperm quality in male fowl. Proc R Soc Lond B 274:853-860

Pizzari T, Worley K, Burke T, Froman D (2008a) Sperm competition dynamics: ejaculate fertilising efficiency changes differentially with time. BMC Evol Biol 8:332

Pizzari T, Dean R, Pacey A, Moore H, Bonsall MB (2008b) The evolutionary ecology of pre- and post-meiotic sperm senescence. Trends Ecol Evol 23:131

Rudolfsen G, Figenschou L, Folstad I, Tveiten H, Figenschou M (2006) Rapid adjustments of sperm characteristics in relation to social status. Proc R Soc Lond B 273:325-332

SAS $^{\circledR}$ (2003) In, vol 9.1, 9.1 edn. SAS Institute, Cary

Stockley P, Gage MJG, Parker GA, Møller AP (1997) Sperm competition in fishes: the evolution of testis size and ejaculate characteristics. Am Nat 149:933-954

Vladić TV, Järvi T (2001) Sperm quality in the alternative reproductive tactics of Atlantic salmon: the importance of the loaded raffle mechanism. Proc R Soc Lond B 268:2375-2381

Wedell N, Gage MJG, Parker GA (2002) Sperm competition, male prudence and sperm-limited females. Trends Ecol Evol 17: 313-320

Wilson-Leedy JG, Ingermann RL (2007) Development of a novel CASA system based on open source software for characterization of zebrafish sperm motility parameters. Theriogenology 67:661-672

Wolfson A (1952) The cloacal protuberance - a means for determining breeding condition in live male passerines. Bird Banding 23:159-165 